\title{
Control of sexually transmitted diseases in the United States-A federal perspective
}

\author{
RALPH H. HENDERSON \\ From the Venereal Disease Control Division, Center for Disease Control, Atlanta, Georgia
}

Introduction

The United States Federal Government has provided grants to states to support venereal disease control programmes since 1939 (Anderson, 1965a). For most of these years, syphilis control was the main objective. In 1972, however, this assistance was increased to $\$ 22.3$ million (more than trebling the annual amounts paid during the previous decade, Fig. 1) and a national gonorrhoea control programme began. Although partly a symptom of changes in the field of venereal disease, the new federal initiative has itself stimulated change, and this article gives a brief overview of the current situation.

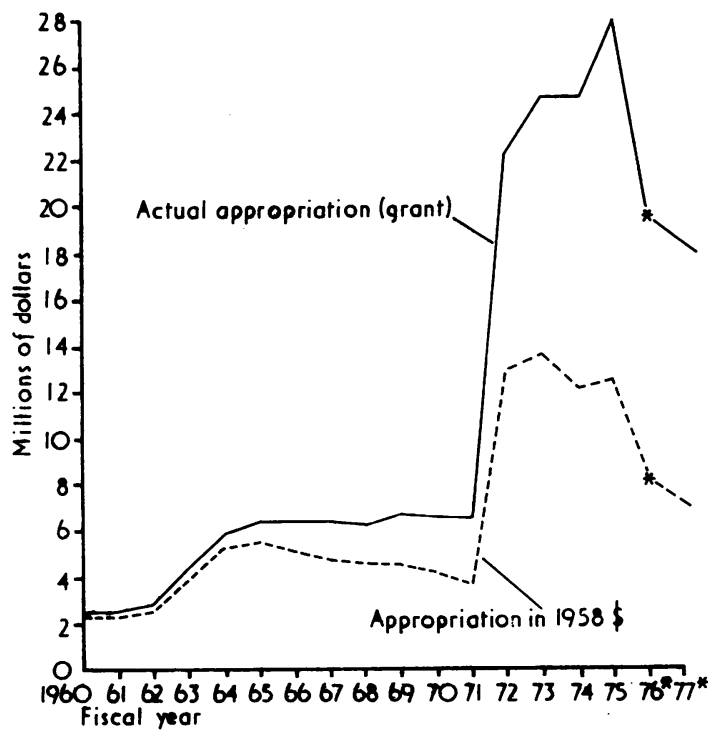

Fig. 1 Actual grant federal appropriation versus federal grant appropriation in 1958 dollars, adjustment for 1958 implicit price deflators for government purchase of goods and services, United States: fiscal years 1960-77

Addrees for reprints: Dr R. H. Henderson, Director, Venereal Disease Control Division, Center for Disease Control, Atlanta, Georgia 30333, USA

Received for publication 15 February 1977
The syphilis era: 1939-71

Anderson reviewed venereal disease control in the United States between 1912 and 1964 (Anderson, 1965a). He described the initiation of the grant in 1939, the establishment of rapid treatment centres for treating syphilis and gonorrhoea during the early 1940s as an emergency wartime measure, and the postwar dismantling of the control apparatus between 1946 and 1955, and this story does not need to be told again here. However, two events should be singled out for special comment. These are the discovery of penicillin as a cure for syphilis, and the postwar decision to terminate direct federal assistance for treating patients with venereal disease. Penicillin left a paradoxical legacy: it works so well that it obviates the need for routine inpatient care, and the dramatic decrease in the incidence of syphilis in the early 1950 s (Fig. 2) which accompanied the widespread use of penicillin convinced many that

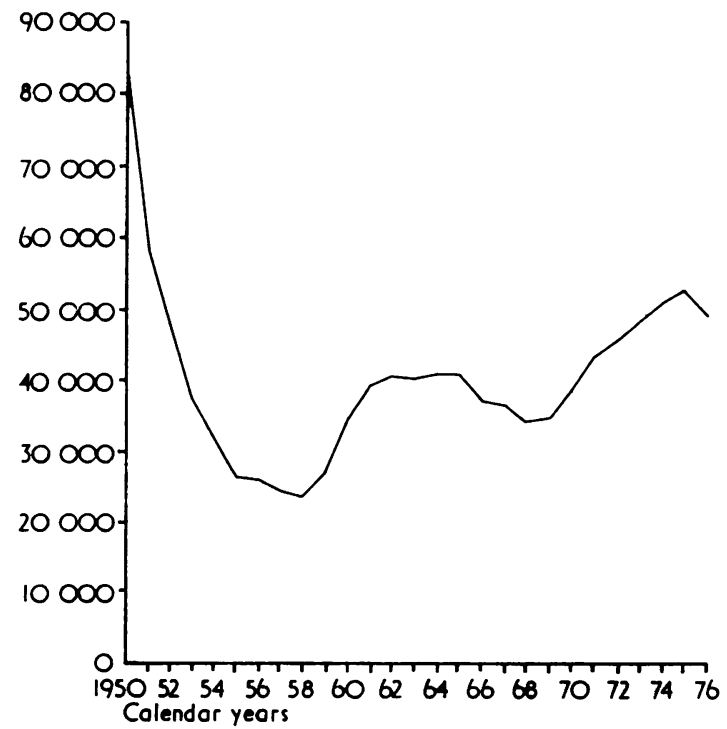

Fig. 2 Early syphilis, United States, calendar years 1950-76 
syphilis eradication was at hand. The speciality of syphilology disappeared. When the rapid treatment centres were closed in the late 1940s and early 1950s by the federal government it was with the agreement of states and the private medical sector, which welcomed federal retreat from an activity which smacked of ' . . f federalised control of medicine and socialisation of medicine' (Anderson, 1965b). The decision came at a time when there appeared to be no future for those practising venereology, and when medical school interest in, and the prestige associated with, this field were sharply declining. The combined impact of these factors has resulted in a neglect of clinical venereology in the United States of America which impairs adequate diagnosis and effective treatment for patients seeking care for sexually transmitted diseases in the public and private sectors.

If the federal attitude of the 1950s was a bane to the venereologist, it was a boon to the venereal disease epidemiologist. Although clinical services were excluded, federal assistance was available to supplement contact tracing services for patients with infectious syphilis. It could also be used to help information, education and serological screening programmes, and to acquire and analyse programme management data. Federal assistance was available in the form of personnel as well as in dollars, and during the 1950s and 1960s the staff of 'public health advisors', working directly at the federal level and as federal assignees to state and local venereal disease control programmes, became a dominant influence.

These staff were recruited from university graduates who served their first years as health department syphilis contact tracers. Capable individuals could gain rapid advancement to the supervisor and managment levels in district, city, county, or state programmes. The experience gained by increasing management responsibility, as well as by multiple geographical assignments within the United States, made these individuals attractive candidates for a variety of venereal disease and other related administrative positions.

The challenge of the assignments and the opportunities for career advancement have attracted and retained a skilled, motivated staff with a high esprit de corps. Their existence has highlighted the epidemiological aspects of venereal disease control programmes. In addition to their work of strengthening all the basic control elements, they have developed standardised techniques for interviewer training, patient and contact interviewing, and for office and field follow-up of patients and contacts. They have also stimulated the development of national information systems which permit contact tracing, screening, and other activities to be monitored at local and national levels.

Although increased federal support for syphilis control in the early 1960 s was followed by a decline in reported early syphilis cases between 1966 and 1968 , the increase was quickly eroded by inflation, and the number of cases began to rise again in 1969 (Figs 1 and 2).

Gonorrhoea and sexually transmitted diseases: 1972-76

In 1964, Thayer and Martin reported the development of a selective culture medium for Neisseria gonorrhoeae and Neisseria meningitidis (Thayer and Martin, 1964). The availability of this new diagnostic technique, coupled with an alarming rise in reported cases of gonorrhoea during the 1960s (Fig. 3), led the federal government in 1968 to invest in six gonorrhoea control pilot projects.* These stressed the importance of widespread casefinding of infected women (using culture screening programmes directed towards women of childbearing age receiving pelvic

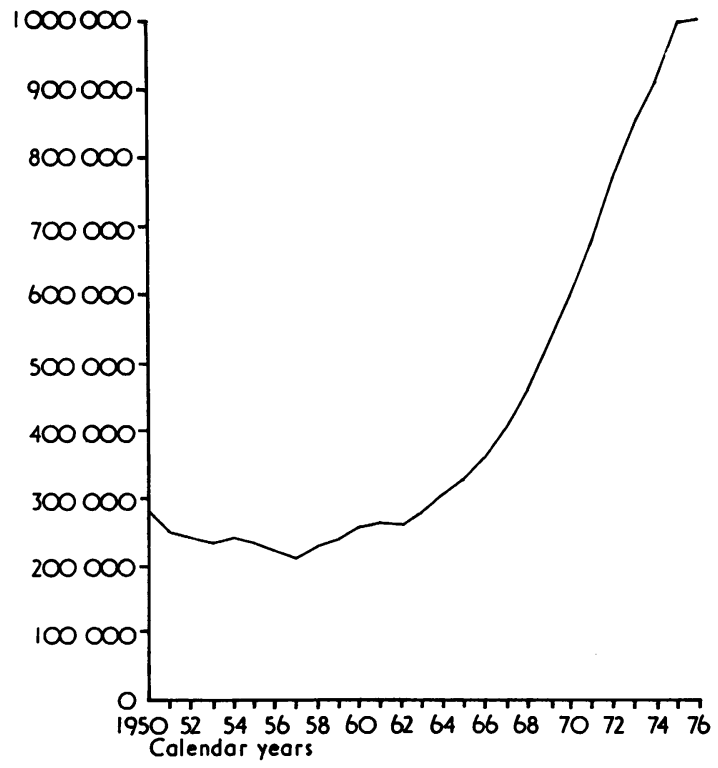

Fig. 3 Gonorrhoea, United States, calendar years 1950-76

*The federal government had experimented with a gonorrhoea control programme in the early 1950 s. In public clinics in 13 cities, men who were diagnosed as having gonorrhoea (often simply because they had a urethral discharge) were interviewed by health workers, and an intensive effort was made to locate and treat their sexual partners. Termed 'speed zone epidemiology', this had been abandoned by 1955 because of lack of funds and lack of demonstrable impact. 
examinations) and the identification and treatment of sexual partners of infected men. Although the effectiveness was not proved, these projects did show the feasibility of such an approach, and the federal government increased its assistance in 1972 to initiate a national gonorrhoea control programme and to stop the increase in infectious syphilis.

From the many changes which accompanied this national commitment to control gonorrhoea, there were tw'o in particular: one concerned the sexually transmitted diseases (STDs) as a group, and the other was a modification of strategies for the prevention of syphilis and gonorrhoea.

Federal concern with gonorrhoea quickly broadened to include other common STDs. Since accurate diagnosis of gonorrhoea is a prerequisite for control, clinics received strong encouragement to provide services for microscopical examination and gonococcal cultures. These services, in turn, improved clinic capacities for diagnosing non-specific urethritis, trichomoniasis, candidiasis, Corynebacterium vaginale vaginitis, and genital herpes infection. Now, adding genital warts, pediculosis, scabies, molluscum contagiosum, and the traditional venereal diseases of syphilis, chancroid, lymphogranuloma venereum, and granuloma inguinale to this list, at least 14 conditions could be diagnosed in an STD clinic.

Improved diagnostic facilities stimulated national concern with the STDs as a group. A second stimulus was the increasing incidence of the diseases themselves (a fact given frequent anecdotal support, but which is only now being documented nationally by prospective data gathered from selected clinics). A third stimulus, however, has resulted from the follow-up and contact referral strategies used with gonorrhoea patients. As opposed to the syphilis programme, in which the public health worker could follow-up each infectious case, gonorrhoea control placed the main burden of compliance on patients and their sex contacts. This made it essential that the clinical services should become responsive to patient needs, and hence be broadened to include the common STDs. Although progress is being made, we are still far short of this goal.

Modifications have been made to both syphilis and gonorrhoea control. Between fiscal years 1969 and 1976, the proportion of men with primary and secondary syphilis who named other men among their sexual partners increased from $24 \%$ to $46 \cdot 3 \%$ (Fig. 4), and, as part of the intensification of syphilis control in 1972, health department activities were directed towards homosexual (gay) men. These included providing syphilis blood testing and gonorrhoea culture screening services in co-operation with bars, bathhouses, and bookstores and supporting clinics (sponsored either by the health department

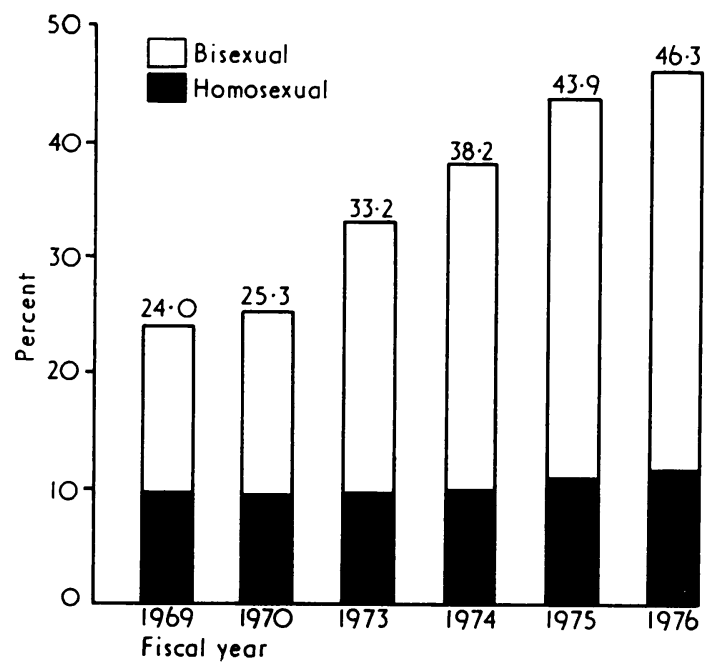

Fig. 4 Primary, secondary, and early (less than one year) latent syphilis; male patients by type of sexual contact, United States: fiscal years 1969-76

or by homosexuals) which were accessible and acceptable to them.

A modification of syphilis interviewing strategies was also introduced during this period. Dubbed 'the lot system', it asked investigators to analyse all epidemiologically linked cases (grouped into one 'lot'), and to determine reinterview and field investigation priorities according to each person's claimed contribution to syphilis transmission as a member of this group. Supervisors are encouraged to assist case workers in the compilation and analysis of these lots. We believe that the combination of improved data with increased supervision has improved the effectiveness of control in towns with a high incidence of syphilis in which this system has been employed.

The modifications to syphilis control were minor, however, compared to those in the gonorrhoea programme. The aim in 1972 was to find asymptomatically infected women, since they were believed to constitute the main disease reservoir leading to the infection and reinfection of men. Men were considered less important disease transmitters since it was felt that most, stimulated by symptoms, would seek early treatment.

By 1975 , this view had changed. It was claimed that most gonorrhoea transmission could be attributed to a few persons (the core) who had several partners and a high prevalence of disease. A proportion of the core was thought to consist of persons who did not suspect they were infected; persons with slight symptoms and signs who had 
not been warned by a sex partner that they might be infected. A larger proportion, however, was thought to consist of persons who knew they might have gonorrhoea, but whose attitudes towards health matters in general led them to do little or nothing about it. These individuals were apt to delay seeking care, and to continue sexual relations in spite of genital symptoms or signs or after being warned by a partner they might be infected. They were apt to comply poorly with treatment and follow-up recommendations, and to take little initiative in helping to ensure that their sexual partners were warned they had been exposed. After treatment, they were soon likely to become reinfected.

The challenge was to design control strategies which permitted a maximum of resources to be concentrated on the core, where the greatest impact on disease transmission was expected. A set of such strategies was described in mid-1975 and circulated to state and local health departments (Henderson, 1975). They recommended that post-treatment cultures (one week after treatment) and re-screening cultures (four to six weeks after treatment) be provided for infected persons, and that priority be given to providing counselling and contact referral services to patients in whom follow-up cultures were positive. A positive culture was interpreted as presumptive evidence that patients and/or their sex partners were members of the core, or that the patients were carrying gonococci which had increased resistance to the antibiotics used. Either interpretation suggested a strong public health rationale for follow-up, reinforcing the recognised benefits from the point of view of the individual's health. In recommending follow-up cultures for men as well as women, and in recommending cultures for all sexual contacts of infected patients, they emphasised the significance of asymptomatic persons of both sexes as gonorrhoea transmitters.

The strategies also included a recommendation for more cultures to be taken from women with pelvic symptoms who presented at outpatient departments (particularly emergency rooms). Since 1972 , it had been known that many cases of gonorrhoea and gonococcal pelvic inflammatory disease were being missed, and that the male partners of such symptomatic women were frequently asymptomatically infected and were therefore likely to be disease transmitters. These strategies confirmed the importance of providing competent and compassionate clinical services.

The trends of both syphilis and gonorrhoea in the United States changed substantially during 1976 (Center for Disease Control, 1977). Early syphilis (primary, secondary, and early latent) declined by $4 \cdot 1 \%$, the first decline since 1967 , while gonorrhoea increased by $0.4 \%$, the smallest increase since 1962 (provisional data).

Because it remains well beyond our capacity to measure the influence of the myriad of factors which affect actual, as well as reported, disease incidence it is not possible to prove that control measures since 1972 have been either a main or a contributory factor associated with these changes. My personal belief is that they have been the dominant factor for both diseases (although less so with gonorrhoea because of its short incubation period and the increased difficulty of reaching and treating exposed sexual partners in time to prevent further spread).

It is also difficult to judge the contribution of each method of control. The impact of the syphilis and gonorrhoea control changes cannot be separated from the impact of changes stimulated by the general increase in public resources devoted to control in 1972. For example, information education programmes and public and professional participation in gonorrhoea screening (in which over nine million cultures were obtained in 1976) have increased awareness of the venereal disease problem in general, and have established the gonococcal culture as an accepted, available, and widely used investigation. Gram stains to differentiate gonococcal from non-gonococcal urethritis in men have also gained wide acceptance. Culture media have improved, as have health department quality control programmes which monitor a variety of elements, including serological testing for syphilis and culturing and Gram staining for gonorrhoea. The revision and dissemination of recommended treatment schedules for gonorrhoea (1972 and 1974) and for syphilis (1976) by the Center for Disease Control have increased the likelihood that patients diagnosed as having these diseases will receive effective treatment. Health departments have expanded by opening new clinics and by extending hours in existing ones; they have also improved the quality of their services.

To estimate the relative importance of these factors, the Venereal Disease Control Division has invested in mathematical modelling to define the theoretical impact of selected intervention programmes, while supporting the establishment of comprehensive data systems in Colombus, Ohio, and Denver, Colorado, to monitor the impact of current programmes.

\section{Future prospects}

Although venereal disease control programmes within the United States are now better established than they had been for many years, much remains to be done. The single greatest impediment to our 
progress is our attitude to this group of diseases. Although this attitude is changing, the reaction of most persons who learn for the first time that they have syphilis or gonorrhoea remains one of shame. Emotionally, these are not perceived as communicable diseases so much as evidence of moral wrong doing. The stigma is powerful, and related to general attitudes toward sexuality.

These attitudes have made it difficult to provide helpful venereal disease education in schools (including schocls for the medical and allied health professions). They have made it difficult to acquire the resources to provide adequate diagriostic and treatment facilities, or to recruit suitatle personnel to staff them. They have made it difficult for patients and their contacts to co-operate fully in the cortact referral process.

The current concern with the troader group of STDs is serving as a welcome countertalance to such attitudes. At present, both the putlic and the medical profession seem to be according an interest in and acceptance of diseases-such as, nonspecific urethritis, genital herpes, and trichomoniasis -which they had leen reluctant to give in the recent past to syphilis and gonorrhoea. Stimulated by increasing incidence, as well as by the increased suppcrt for STD research and fellowships which has been made available from the National Institutes of Health, doctors from a variety of specialties (including internal medicine, infectious disease, obstetrics and gynaecology, and urology) have joined the dermatologists in contributing to research, teaching, and patient care in this field, and this trend is expected to continue and to contribute to improved medical training. Significant advances have been made in understanding the biology of the gonccoccus, and of the immunology and epidemiology of gonococcal infections, so much so that gonorrhoea even threatens to become fashionable as a teaching and research model.

An eloquent witness to the resurgence of interest in the sexually transmitted diseases in the United States has been the rebirth of an American journal devoted to this ficld. The Journal of the American Venereal Disease Association* published its first issue in September 1974, 20 years after the appearance of the last issue of the American Journal of Syphilis, Gonorrhea, and Venereal Diseases, and is beginning to fill the void left by its predecessor.

\footnotetext{
* Now named Sexually Transmitted Diseases
}

Our problems with attitudes are accompanied by other difficulties. Of immediate concern is the level of support teing provided by the federal government. The effects of inflation, coupled with reductions in actual appropriations, reduced fiscal year 1977 federal dollar purchasing power by $45 \%$ compared with 1972 (Fig. 1). A second problem, the dimensions of which are still being defined but which promises to be both serious and on a large scale, is the emergence of the penicillinase-producing strains of Neisseria gonorrhoeae.

Eventually, we may find a variety of preventive community health services which now often receive federal support (such as, venereal disease, immunisation, family planning, maternal child health, and environmental health services) becoming integrated into broadened health care planning and national health insurance programmes. This may entail a transition period of uncertainty (and perhaps weakened impact) during which issues of payment sources and mechanisms, authority and accountability for operations, and the nature of the programmes are resolved.

A lthough research discoveries, continued changes in sexual behaviour, and changes in the infectious agents themselves will undoubtedly alter the specific content of STD control programmes, it seems certain that this group of diseases will continue to pose significant national and international health threats for the foreseeable future. The Venereal Disease Control Division's most challenging tasks in coming years will be to foster the development of more constructive public and professional attitudes toward the STDs, and to contribute to national health policy formulation to assist the United States to improve the calibre of its STD clinical services, while retaining its strength with respect to STD epidemiological services and research.

\section{References}

Anderson, O. W. (1965a). Syphilis and Society-Problems of Control in the United States 1912-1964, p. 10. University of Chicago: Illinois. Anderson, O. W. (1965b). Extract from House Appropriations Committee on Appropriations Bill for 1947, pp. 16-17. Quoted in Syphilis and Society-Problems of Control in the United States 1912-1964, p. 22. University of Chicago: Illinois.

Center for Disease Control (1977). Gonorrhea and early syphilis cases -United States, 1976. Morbidity and Mortality Weekly Report, 26, 1 .

Henderson, R. H. (1975). Commentary on National Strategies to Control Gonorrhea. US Department of Health, Education, and Welfare: Georgia.

Thayer, J. D., and Martin, J. E. Jr (1964). A selective medium for the cultivation of $N$. gonorrhoeae and $N$. meningitidis. Public Health Reports, 79, 49-57. 